\title{
Über den Einfluss der Volumveränderungen der Blutfliussigkeit auf die zirkulierende Blutmenge im normalen und pathologischen Zustand.
}

\author{
IV. Mitteilung: Die Veränderungen der zirkulierenden Blutmenge \\ durch Bluttransfusion bei gesunden sowie Kantharidintieren.
}

\author{
Von \\ Seiichi Takahashi. \\ (高 橋 濟一) \\ (Aus der Medizinischen Klinik von Prof. Dr. T. Kato, \\ Tohoku Reichsuniversität zu Sendci.)
}

Die klinisch und therapeutisch höchst wichtige Bedeutung muss einer kritischen Bewertung beschieden sein, welche Verschiebung die zirkulierende Blutmenge durch die Bluttransfusion erleidet. Denn an die Verhältnisse, wozu die Bluttransfusion erforderlich ist, ist die Kreislaufschwäche irgendwelcher Art fast stets angeknüpft. Bei der Durchsicht der einschlägigen Literatur fällt es uns besonders auf, dass unter den Anschauungen von verschiedenen Autoren über diesen Gegenstand manchmal Widersprüche auftauchen.

So z. B. haben Boycott und Douglas, ${ }^{1)}$ Krumbhaar und Channtin'? eine Zunahme der zirkulierenden Blu tmenge durch die Transfusion gesehen, $\mathrm{F} u$ ruhas $\mathrm{i}^{3}$ hat hingegen nachgewiesen, dass die zirkulierende Blutmenge bald nach Bluttransfusion eine geringe Zunahme aufweist, nach 4 Stunden umgekehrt ein wenig abnimmt. Nachdem B oycott und Oakler) nach Bluttransfusion die Vermehrung der zirkulierenden Blutmenge festgestellt hatten, haben sie weiterhin die Bestimmung der Gesamtblutmenge nach der Welckers Methode durchgeführt und kamen zum Ergebnis, dass die Plasmamenge zunächst zunimmt, nach 2 oder 3 Tagen aber auf den früheren Niveau zurückkommt, wäh-

1) Boycott and Douglas, Journ. of Patholog. and Bacteriol, 1909, 13, 418 and $1910,14,294$.

2) Krumbhaar and Channtin, Journ. of Exp. Med., 1921, 35, 848.

3) Furuhashi, Aichi Igakkai Zasshi, 1929, 36, 1707.

4) Boycott and $\mathrm{Oakler}$, Jonrn. of Pathol. and Bacteriol., 1933, 36, 205 and 1934, 38, 91 . 
rend das Erythrozytenvolum hingegen seinen einmal erhöhten Wert weiter beibehält. Doch fehlt es nicht an Autoren, welche gegen die Annahme der Vermehrung der zirkulierenden Blutmenge Protest erheben, unter anderem behauptet der Autor, wie $\mathrm{Ke} \mathrm{it} h,{ }^{5)}$ dass die zirkulierende Blutmenge durch die Bluttransfusion keineswegs zunehme, indem das transfundierte Blut oder die demselben entsprechende Blutmenge sofort nach Bluttransfusion in Blutdepots reserviert werde.

Über die Veränderung der zirkulierenden Blutmenge nach Bluttransfusion gelangt man, wie oben auseinandergesetzt, noch nicht zu endgültiger Ansicht. Es ist von vornherein leicht verständlich, dass da, wo infolge von Schädigungen der Gefässe bzw. Gewebe funktionelle Veränderungen derselben sich einstellen und hieraus Störungen in Flüssigkeitsaustausch zwischen Blut und Gewebe ausgelöst worden sind, oben angeführte Veränderungen der zirkulierenden Blutmenge durch Bluttransfusion sich anders verhalten müssen; und doch sind die daraufhin gerichteten Untersuchungen meines Wissens bis dahin noch kaum ausgeführ't worden. In dieser Eı wägung wurde in vorliegender Versuchsreihe zunächst bei gesunden Kaninchen dieBluttransfusion vorgenommen und daraus resultierende Verschiebung der zirkulierenden Blutmenge verfolgt, während man aus den gleichzeitig. damit ermittelten Veränderungen der Plasmamenge und des Erythrozytenvolums einen Einblick in den Mechanismus der Verschiebung der zirkulierenden Blutmenge zu gewinnen versuchte. Demnächst wurde an Kaninchen, deren Gewebe und Gefässwand auf ganz gleiche Weise wie im in den vorangehenden Mitteilungen ${ }^{6 / i)}$ erwähnten Experiment mit Kantharidin geschädigt waren und bei denen die eben dieselbe Bluttransfusion vorgenommen wurde, die Paralleluntersuchungen angestellt.

Versuchsmethodik: Es wurden dem Blut, welches einem gesunden Kaninchen durch den Aderlass rasch entnommen wurde, eine 2,5\% Natriumcitratlösung im Mengenverhältnis von $10 \%$ zugesetzt, hier wurde also ein $0,25 \%$ iges Natriumcitrat-Blutgemisch hergestellt. Nachdem man Kaninchen von diesem Gemisch $20 \mathrm{ccm}$ pro kg transfundiert hatte, wurden die Messungen von zirkulierender Blutmenge, Plasmamenge und Erythrozytenvolum vorgenommen. Diese Messungen wurden eine Stunde vor der Bluttransfusion, dann in direktem Anschluss daran und eine und zwei Stunden danach, in manchen Fällen

5) Ke ith, Medical Research Council, 1919, Special Report: zit.nach A sh by, Arch. of Int. Med., 1925, 35, 641.

6) Takahashi, Tohoku Journ. Exp. Med., 1935, 25, 531.

7) Takahashi, Tohoku Journ. Exp. Med., 1935, 25, 550. 
auch drei Stunden später durchgeführt. Die Bestimmung der zirkulierenden Blutmenge geschah nach der von Seyderhelm und $\mathrm{Lampe} \mathrm{e}^{\mathrm{s})}$ angegebenen Trypanrotmethode, wie sie auch im Versuch in erster Mitteilung ${ }^{6)}$ herangezogen wurde.

\section{Versuch an gesunden Kaninchen.}

Ist bei 5 gesunden Kaninchen die Bluttransfusion ron $20 \mathrm{ccm}$ pro $\mathrm{kg}$ durchgeführt worden, dann weist die zirkulierende Blutmenge unmittelbar danach in allen Fällen eine Zunahme um durchschnittlich $18,1 \%(8,7-24,9 \%)$ auf (Tab. 1). Dieser Betrag der vermehrten Blutmenge war aber weniger als die Menge des Transfundates. Es ist dies höchstwahrscheinlich darauf zurückzuführen, dass die Blutbahn, weil es hierbei durch die Bluttransfusion zum Überschuss an Blut kommt, einen Teil von Blut in die Blutdepots hineinzwängt.

Bei früher vorgenommenen Infusionen von physiologischer Kochsalzlösung wie auch von der Gummilösung hat man sich davon überzeugt, dass die zirkulierende Blutmenge unmittelbar nach Infusionen nicht zunimmt, sondern umgekehrt ein wenig abnimmt. Dabei wurde hervorgehoben, dass der Hauptfaktor hierfür darin zu suchen ist, dass bei Infusionen derartiger Lösungen die Blutdepots, insbesondere aber die Gefässprovinzen im Splanchnicusgebiet durch überschüssige Salzoder Gummilösung auf nervösem Wege reflektorisch übermässig dilatiert werden, und ferner dass die damit einhergehende geringfügige Verengerung der allgemeinen Gefässbahn als Hilfsursache eine Rolle spielen muss. Aus vorliegenden Untersuchungen hat es sich indessen, wie oben geschildert, herausgestellt, dass die zirkulierende Blutmenge nach Bluttransfusion merklich zunimmt. Demnach dürfte angenommen werden, dass das transfundierte Blut nach seinem Eintritt in die Blutbahn, ohne die Blutdepots stark zu reizen und auch das Gefässsystem überhaupt funktionell wesentlich zu beeinflussen, mithin ohne auch in den Blutdepots in grösseren Mengen zurückgehalten zu werden, gleichsam wie es gewesen ist, ins Zirkulationssystem des Empfängers aufgenommen zu werden und darin zu zirkulieren vermag.

Die zirkulierende Blutmenge, welche nach transitorischer $\mathrm{Zu}$ nahme allmählich abzunehmen anfängt, zeigt in einstündigem Ablauf nach Bluttransfusion Neigung zu dem Niveau vor der Transfusion und dennoch eine Zunahme um durchschnittlich $8,7 \%(3,9-12,0)$ gegen-

8) Seyderhel m und L a m pe, Erg. d. inn. Med. und Kinderheilk., 1925, 27, 245. 
über dem Vorwert. Nach 2 Stunden wies sie in den sämtlichen Fällen noch eine Zunahme, welche gegenüber dem Vorwert 9,6\% (5,4-14,8\%) betrug. Nach 3 Stunden war sie in 3 Fällen (Versuch 1,4 und 5) höher gegenüber dem Vorwert, während sie in 2 Fällen (Versuch 2 und 3) auf den annähernden Vorwert zurückkam.

Die Plasmamenge stieg unmittelbar nach der Bluttransfusion in den sämtlichen Fällen über die Anfangsgrösse hinaus, indem sie im Durchschnitt eine Zunahme von 17,1\% (10,5-20,7\%) zeigte. Nach

Tabelle 1.

Veränderung der Blutmenge nach Bluttransfusion bei normalen Kaninchen.

\begin{tabular}{|c|c|c|c|c|c|c|c|c|c|c|c|c|c|}
\hline \multirow{2}{*}{$\dot{\bar{z}}$} & \multirow{2}{*}{ 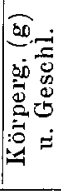 } & \multirow{2}{*}{$\begin{array}{l}\text { Zeit d. } \\
\text { Blutmengen- } \\
\text { bestimmung }\end{array}$} & \multirow{2}{*}{ 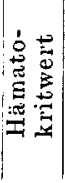 } & \multicolumn{3}{|c|}{$\begin{array}{l}\text { Zirkulierende } \\
\text { Blutmenge }\end{array}$} & \multicolumn{3}{|c|}{ Plasmamenge } & \multicolumn{3}{|c|}{$\begin{array}{c}\text { Erythrozyten- } \\
\text { volum }\end{array}$} & \multirow{2}{*}{ 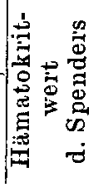 } \\
\hline & & & & 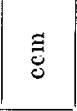 & 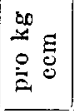 & $\begin{array}{l} \pm \\
0 \\
0\end{array}$ & $\Xi$ & $\stackrel{30}{20}$ & : & छ & 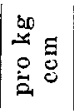 & $\begin{array}{l}z \\
0 \\
0\end{array}$ & \\
\hline 1 & $\begin{array}{c}2000 \\
\delta\end{array}$ & 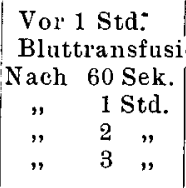 & $\begin{array}{l}48,0 \\
\text { on }(4 \\
47,3 \\
48,0 \\
46,3 \\
46,7\end{array}$ & $\begin{array}{l}146,9 \\
0 \mathrm{ccm} \\
160,1 \\
160,9 \\
167,9 \\
162,7\end{array}$ & $\begin{array}{r}73,4 \\
\left.\text { in } 2^{\prime}\right) \\
80,0 \\
80,4 \\
83,9 \\
81,3\end{array}$ & $\begin{array}{r}+8,9 \\
+\quad 9,5 \\
+14,3 \\
+10,7\end{array}$ & $\begin{array}{l}84,4 \\
83,7 \\
90,2 \\
86.7\end{array}$ & $\begin{array}{l}38,2 \\
42,2 \\
41,8 \\
45,1 \\
43,3\end{array}$ & $\begin{array}{r}+10,5 \\
+9,5 \\
+18,1 \\
+13,5\end{array}$ & $\begin{array}{l}70,5 \\
75,7 \\
77,2 \\
77,7 \\
75,9\end{array}$ & $\begin{array}{l}35,2 \\
\\
37,8 \\
38,6 \\
38,8 \\
37,9\end{array}$ & $\begin{array}{r}+\quad 7,4 \\
+\quad 9,5 \\
+10,2 \\
+\quad 7,6\end{array}$ & 47 \\
\hline 2 & $\begin{array}{c}1600 \\
5\end{array}$ & 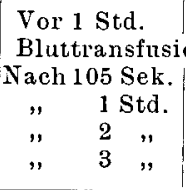 & $\begin{array}{l}51,7 \\
\text { on }(3 \\
52,3 \\
55,7 \\
54,3 \\
55,2\end{array}$ & $\begin{array}{l}107,8 \\
2 \mathrm{ccm} \\
130,4 \\
120,7 \\
116,3 \\
108,6\end{array}$ & $\begin{array}{r}67,4 \\
\text { in } 60 \prime \prime \\
81,5 \\
75,4 \\
72,7 \\
67,9\end{array}$ & $\begin{array}{l}\text { "I) } \\
+21,0 \\
+12,0 \\
+7,9 \\
+0,7\end{array}$ & $\begin{array}{l}62,2 \\
53,5 \\
53,1 \\
48,7\end{array}$ & $\begin{array}{l}32,6 \\
38,9 \\
33,4 \\
33,2 \\
30,4\end{array}$ & $\begin{array}{r}+19,4 \\
+\quad 2,7 \\
+\quad 1,9 \\
-\quad 8,4\end{array}$ & $\begin{array}{l}55,7 \\
68,2 \\
67,2 \\
63,2 \\
59,9\end{array}$ & $\begin{array}{l}34,8 \\
42,6 \\
42,0 \\
39,5 \\
37,4\end{array}$ & $\begin{array}{r}+22,4 \\
+20,6 \\
+13,5 \\
+\quad 7,5\end{array}$ & 48 \\
\hline 3 & $\left|\begin{array}{c}1820 \\
6\end{array}\right|$ & 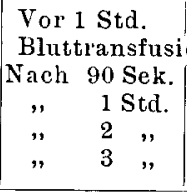 & $\begin{array}{l}43,0 \\
\text { on }(3 \\
45,3 \\
46,8 \\
46,0 \\
45,1\end{array}$ & $\begin{array}{l}126,8 \\
6 \mathrm{ccm} \\
157,6 \\
139,1 \\
145,6 \\
123,5\end{array}$ & $\begin{array}{r}69,7 \\
\text { in } 65 \prime \prime \\
86,6 \\
76,4 \\
80,0 \\
67,8\end{array}$ & $\begin{array}{l}\text { "I) } \\
+24,3 \\
+9,7 \\
+14,8 \\
-\quad 2,6\end{array}$ & $\begin{array}{l}72,3 \\
86,1 \\
74,0 \\
78,6 \\
67,8\end{array}$ & $\begin{array}{l}39,7 \\
47,3 \\
40,6 \\
43,2 \\
37,2\end{array}$ & $\begin{array}{r}+19,1 \\
+\quad 2,3 \\
+\quad 8,7 \\
-\quad 6,2\end{array}$ & $\begin{array}{l}54,5 \\
71,5 \\
65,1 \\
67,0 \\
\mathbf{5 5 , 7}\end{array}$ & \begin{tabular}{l|}
30,0 \\
\\
39,3 \\
35,8 \\
36,8 \\
30,6
\end{tabular} & $\begin{array}{r}+31,2 \\
+19,4 \\
+22,9 \\
+2,2\end{array}$ & 44 \\
\hline 4 & 2240 & 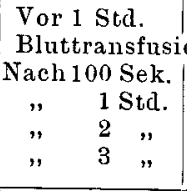 & $\begin{array}{l}46,0 \\
\text { on }(4 \\
47,6 \\
52,0 \\
49,0 \\
48,5\end{array}$ & $\begin{array}{c}141,1 \\
5 \mathrm{~cm} \\
176,3 \\
153,3 \\
148,8 \\
152,0\end{array}$ & $\begin{array}{r}63,0 \\
\text { in } 65 \\
78,7 \\
68,4 \\
66,4 \\
67,8\end{array}$ & $\begin{array}{r}\prime) \\
+24,9 \\
+\quad 8,6 \\
+\quad 5,4 \\
+\quad 7,7\end{array}$ & $\begin{array}{l}92,4 \\
73,7 \\
75,9 \\
78,3\end{array}$ & $\begin{array}{l}51,2 \\
32,9 \\
33,9 \\
34,9\end{array}$ & $\begin{array}{r}+20,7 \\
-3,3 \\
-\quad 0,4 \\
+\quad 2,7\end{array}$ & \begin{tabular}{l|}
64,9 \\
\\
83,9 \\
79,6 \\
72,9 \\
73,7
\end{tabular} & $\begin{array}{l}29,0 \\
37,4 \\
35,5 \\
32,5 \\
32,9\end{array}$ & $\begin{array}{r}+29,3 \\
+22,6 \\
+12,3 \\
+13,6\end{array}$ & 48 \\
\hline 5 & 1950 & 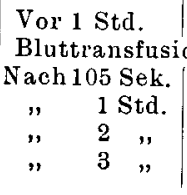 & $\begin{array}{l}\mid 47,3 \\
\text { on }(4 \\
45,3 \\
49,6 \\
50,5 \\
48,6\end{array}$ & $\begin{array}{l}149,0 \\
0 \mathrm{ccm} \\
166,3 \\
154,9 \\
157,1 \\
154,6\end{array}$ & $\begin{array}{r}76,4 \\
\text { in } 55 \prime \prime \\
85,3 \\
79,4 \\
80,6 \\
79,3\end{array}$ & $\begin{array}{l}\text { ") } \\
+11,6 \\
+\quad 3,9 \\
+\quad 5,4 \\
+\quad 3,7\end{array}$ & $\begin{array}{l}91,0 \\
78,1 \\
77,8 \\
79,5\end{array}$ & $\begin{array}{l}46,7 \\
40,0 \\
39,9 \\
40,8\end{array}$ & $\begin{array}{r}+15,8 \\
-\quad 0,6 \\
-\quad 1,0 \\
+\quad 1,1\end{array}$ & $\begin{array}{l}75,3 \\
76,8 \\
79,3 \\
75,1\end{array}$ & $\begin{array}{l}36,1 \\
38,6 \\
39,4 \\
40,7 \\
38,5\end{array}$ & $\begin{array}{l}+\quad 6,4 \\
+\quad 9,1 \\
+12,6 \\
+\quad 6,7\end{array}$ & 45 \\
\hline
\end{tabular}


Fig. 1. Veränderung der zirkulierenden Blutmenge nach Bluttransfusion bei gesunden Kaninchen (Versuch 2).

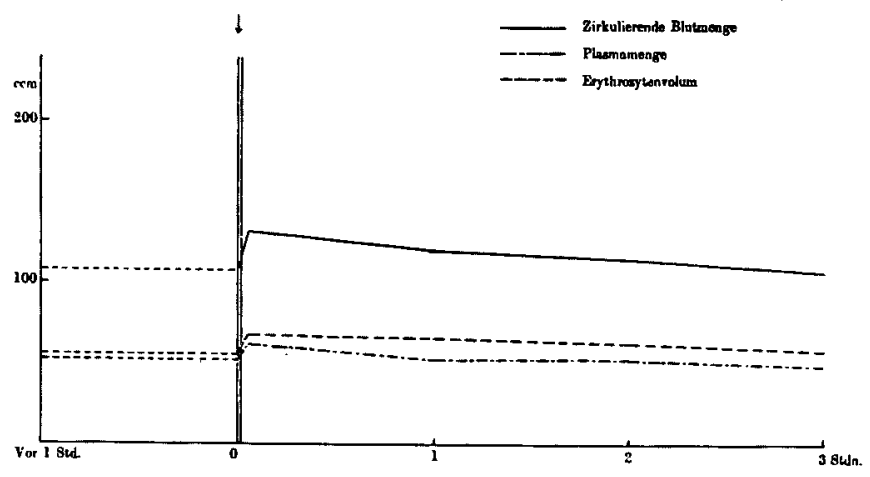

einer Stunde rückte sie in 4 Fällen (Versuch 2, 3,4 und 5) schon an den Vorwert heran, nur in einem einzigen Fall (Versuch 1) wies sie noch einen höheren Wert als der Vorwert auf. Nach 2 Stunden nahm die Plasmamenge in 2 Fällen (Versuch 1 und 2) ein wenig mehr zu als nach einstündigem Ablauf, während sie in anderen 3 Fällen (Versuch 2, 4 und 5) an den annähernden Vorwert herantrat. Nach 3 Stunden nahm sie in einem einzigen Fall (Versuch 1) um ein geringes mehr zu, als vor der Bluttransfusion, während sie in anderen 4 Fällen entweder den annähernden Vorwert erreichte oder darunter absank.

Das Erythrozytenvolum zeigte gleichnach der Bluttransfusion eine Zunahme um durchschnittlich 19,3\% (6,4-31,2\%), welche also grösser war als die der Plasmamenge in derselben Phase. Die geringere Zunahme der Plasmamenge beruht offensichtlich darauf, dass die Plasmaflüssigkeit im Laufe der Transfusion gewissermassen durch die Blutbahn hindurchgesickert ist. Darauf, mit dem Zeitablauf zeigte das Erythrozytenvolum immer stärkere Zuneigung zu früherem Niveau, nach einem-, zwei- und dreistündigem Ablauf wurden im Durchschnitt dennoch Zunahmen resp. um 16,2\% (9,1-22,6\%), um 14,3\% $(10,2-22,9 \%)$ und um $7,5 \%(2,2-13,6 \%)$ gegenüber dem Vorwert ermittelt. Hieraus geht also hervor, dass das Erythrozytenvolum in dreistündigem Ablauf nach Transfusion noch einen höheren Wert aufweist als vor der Bluttransfusion.

Aus obigen Ergebnissen hat es sich nämlich herausgestellt, dass, wenn bei gesunden Kaninchen die Bluttransfusion durchgeführt worden ist, die zirkulierende Blutmenge unmittelbar danach deutlich zunimmt, bald darauf aber allmählich an den Vorwert herantritt, so dass 
sie nach 3 Stunden in wenigen Fällen auf den Vorwert zurückkommt. Und wenn mir einen Rückblick auf die Beziehung zwischen der Plasmamenge und dem Erythrozytenvolum werfen, so springt es in die Augen, dass die Plasmamenge schon eine Stunde nach der Bluttransfusion in der Mehrzahl der Fälle auf den Vorwert zurückkommt, während das Erythrozytenvolum hingegen selbst in dreistündigem Ablauf noch höhere Werte als der Vorwert aufweist. Ein derartiges Phänomen tritt bei Infusionen von der Kochsalz- oder Gummilösung niemals in Erscheinung und spricht offenbar dafür, dass der Transfusion des sog. nativen Blutes die biologisch zweckmässigere Bedeutung als den Infusionen von künstlich hergestellter NaCl-Lösung oder Gummilösung zufällt.

\section{Versuch an Kantharidinkaninchen.}

In vorangehender Versuchsreihe wurde festgestellt, dass die durch Bluttransfusion vermehrte Blutmenge in erster Linie durch $\mathrm{Ab}$ nahme der Plasmamenge auf den Wert vor der Transfusion zurückkommt. Aus dieser Feststellung erhellt also, dass der Verlauf der nach Bluttransfusion einsetzenden Veränderungen der Blutmenge vor allen Dingen mit dem Wasseraustausch zwischen Gefässen und Geweben aufs innigste zusammenhängt. Von diesem Gedankengang ausgehend, wurde in vorliegender Versuchsreihe bei denjenigen Kaninchen, deren Wasseraustausch im Gewebe durch Vergiftung mit Kantharidin gestört war, die Bluttransfusion durchgeführ't und der Verlauf der dadurch auftretenden Blutmengenänderungen beobachtet.

Es wurden 4 gesunden Kaniuchen 0,5 mg Kantharidin pro kg injiziert, und nachdem durch die 2+ Stınden später vorgenommene Harnuntersuchung das Auftreten der Vergiftungssymptome sichergestellt worden war, wurde ihnen vom Blut, welches auf ähnliche Weise wie bei gesunden Kaninchen entnommen wurde, $20 \mathrm{ccm}$ pro $\mathrm{kg}$ transfundiert (Tab. 2).

Die zirkulierende Blutmenge in der Bluttransfusion unmittelbar nachfolgender Zeit wies in den sämtlichen Fällen eine Zunahme auf, welche im Durchschnitt 8,9\% (0,9-20,4\%) betrug. Diese Zunahme war also geringer als die bei gesunden Kaninchen. Sie war auch spärlicher im Vergleich zu durchschnittlicher Zunahme von $11,3 \%$, welche bei Kantharidinkaninchen (in zweiter Mitteilung) ${ }^{7)}$ in der Gummiinfusion unmittelbar nachfolgender Zeit ermittelt wurde. Die zirkulierende Blutmenge nahm nach einer Stunde allmählich ab.

Die Intensität dieser Abnahme entsprach im grossen und ganzen dem Harnbefund. In Versuch 2 nämlich, in dem das Harneiweiss sich als am geringsten 
Tabelle 2.

Veränderung der Blutmenge nach Bluttransfusion bei Kantharidinkaninchen.

\begin{tabular}{|c|c|c|c|c|c|c|c|c|c|c|c|c|c|}
\hline \multirow{2}{*}{$\dot{\dot{z}}$} & \multirow{2}{*}{ 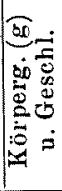 } & \multirow{2}{*}{$\begin{array}{l}\text { Zeit d. } \\
\text { Blutmengen- } \\
\text { bestimmung }\end{array}$} & \multirow{2}{*}{ 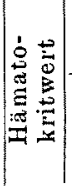 } & \multicolumn{3}{|c|}{$\begin{array}{l}\text { Zirkulierende } \\
\text { Blutmenge }\end{array}$} & \multicolumn{3}{|c|}{ Plasmamenge } & \multicolumn{3}{|c|}{$\begin{array}{c}\text { Erythrozyten- } \\
\text { volum }\end{array}$} & \multirow{2}{*}{ 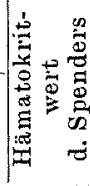 } \\
\hline & & & & క్ & 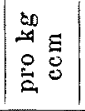 & $\begin{array}{l} \pm \\
40 \\
0\end{array}$ & $\stackrel{g}{8}$ & $\begin{array}{l}x_{0} \\
-1 \\
0 \\
0 \\
\Xi\end{array}$ & 혐 & 罗 & $\begin{array}{l}\infty 0 \\
0 \\
0 \\
0\end{array}$ & $\begin{array}{l}\exists \\
\nexists 2\end{array}$ & \\
\hline 1 & $\mid \begin{array}{c}2040 \\
\delta\end{array}$ & $\begin{array}{l}\text { Vor } 1 \text { Std. } \\
\text { Bluttransfusi } \\
\text { Näch } 60 \text { Sek. } \\
\quad \text { " } 1 \text { Std. } \\
\quad \text { " } 2 \text { " }\end{array}$ & $\begin{array}{l}\mid \begin{array}{r}41,2 \\
\text { ion }(4 \\
\left|\begin{array}{l}43,6 \\
44,6 \\
44,0\end{array}\right|\end{array}\end{array}$ & $\begin{array}{l}175,8 \\
0 \mathrm{~cm} \\
188,1 \\
162,5 \\
152,8\end{array}$ & $\begin{array}{r}86,2 \\
\text { in } 45 \\
92,2 \\
79,6 \\
74,9\end{array}$ & $\begin{array}{l} \\
+7,0 \\
-7,6 \\
-13,1\end{array}$ & $\begin{array}{r}103,4 \\
106,6 \\
84,5 \\
74,4\end{array}$ & $\begin{array}{l}50,7 \\
52,2 \\
41,4 \\
36,5\end{array}$ & $\begin{array}{r}+3,1 \\
-18,3 \\
-28,0\end{array}$ & $\begin{array}{l}72,4 \\
\\
81,5 \\
78,0 \\
78,4\end{array}$ & $\begin{array}{l}35,5 \\
\\
39,9 \\
38,2 \\
38,4\end{array}$ & $\begin{array}{r}+12,6 \\
+\quad 7,7 \\
+\quad 8,3\end{array}$ & 45 \\
\hline 2 & $\begin{array}{c}1950 \\
0\end{array} \mid$ & $\begin{array}{l}\text { Vor } 1 \text { Std. } \\
\text { Bluttransfusi } \\
\text { Nach } 60 \text { Sek. } \\
\quad " \quad 1 \text { Std. } \\
\quad " \quad 2 \text { " }\end{array}$ & $\begin{array}{l}45,0 \\
\text { ion }(4 \\
49,0 \\
50,0 \\
51,0\end{array}$ & $\begin{array}{l}117,8 \\
0 \mathrm{ccm} \\
141,9 \\
141,0 \\
137,5\end{array}$ & $\begin{array}{l}60,4 \\
\text { in } 1 \text { ') } \\
72,8 \\
72,3 \\
70,5\end{array}$ & $\begin{array}{l}+20,4 \\
+19,7 \\
+16,7\end{array}$ & $\begin{array}{l}72,4 \\
70,5 \\
67,4\end{array}$ & $\begin{array}{l}33,2 \\
\\
37,1 \\
36,1 \\
34,6\end{array}$ & $\begin{array}{r}+11,7 \\
+\quad 8,9 \\
+\quad 4,0\end{array}$ & $\begin{array}{l}53,0 \\
69,5 \\
70,5 \\
70,1\end{array}$ & $\begin{array}{l}27,2 \\
\\
35,6 \\
36,1 \\
35,9\end{array}$ & $\begin{array}{l}+31,1 \\
+33,0 \\
+32,3\end{array}$ & 49 \\
\hline 3 & $\begin{array}{c}1660 \\
6\end{array}$ & $\begin{array}{l}\text { Vor } 1 \text { Std. } \\
\text { Bluttransfusi } \\
\text { Nach } 80 \text { Sek. } \\
\quad " \quad 1 \text { Std. } \\
\quad " \quad 2 \quad,\end{array}$ & $\begin{array}{r}\mid \begin{array}{r}44,6 \\
\text { ion }(3 \\
43,5 \\
44,6 \\
45,0\end{array}\end{array}$ & $\begin{array}{c}131,6 \\
0 \mathrm{ccm} \\
132,8 \\
125,9 \\
123,5\end{array}$ & \begin{tabular}{|c|}
79,2 \\
in $40^{\prime \prime}$ \\
80,0 \\
75,8 \\
74,4
\end{tabular} \mid & $\begin{array}{l}1 / 1 \\
+\quad 0,9 \\
-\quad 4,2 \\
-\quad 5,9\end{array}$ & $\begin{array}{l}73,9 \\
69,7 \\
67,9\end{array}$ & $\begin{array}{l}44,5 \\
42,0 \\
40,9\end{array}$ & $\begin{array}{r}+\quad 0,1 \\
-\quad 0,4 \\
-\quad 0,7\end{array}$ & $\begin{array}{l}58,9 \\
56,1 \\
55,6\end{array}$ & $\begin{array}{l}35,4 \\
\\
35,5 \\
33,8 \\
33,5\end{array}$ & $\begin{array}{l}+0,3 \\
-4,4 \\
-3,6\end{array}$ & 40 \\
\hline 4 & $=\begin{array}{c}1790 \\
0\end{array}$ & $\begin{array}{l}\text { Vor } 1 \text { Std. } \\
\text { Bluttransfusi } \\
\text { Nach } 60 \text { Sek. } \\
\quad " \quad 1 \text { Std. } \\
\quad " \quad 2 \text { " }\end{array}$ & $\begin{array}{l}51,0 \\
\text { ion }(3 \\
\qquad \begin{array}{l}48,0 \\
51,0 \\
51,0\end{array} \mid\end{array}$ & $\begin{array}{l}140,6 \\
6 \mathrm{ccm} \\
150,9 \\
143,1 \\
137,1\end{array}$ & \begin{tabular}{|c|}
78,5 \\
in $2^{\prime}$ ) \\
$9 \begin{array}{c}84,3 \\
79,9 \\
76,6\end{array} \mid$
\end{tabular} & $\begin{array}{r} \\
+\quad 7,3 \\
+\quad 1,8 \\
-2,5\end{array}$ & $\begin{array}{l}78,5 \\
70,1 \\
67,2\end{array}$ & $\begin{array}{l}43,8 \\
39,1 \\
37,5\end{array}$ & $\begin{array}{r}+13,9 \\
+\quad 1,7 \\
-\quad 2,5\end{array}$ & $\begin{array}{l}71,7 \\
72,4 \\
73,0 \\
69,9\end{array}$ & $\begin{array}{l}40,0 \\
40,4 \\
40,8 \\
39,0\end{array}$ & $\begin{array}{r}+\quad 1,0 \\
+\quad 1,8 \\
-\quad 2,5\end{array}$ & 48 \\
\hline
\end{tabular}

erwies, zeigte die zirkulierende Blutmenge an diesem Zeitpunkt keinen merklichen Unterschied gegenüber dem unmittelbar nach der Bluttransfusion ermittelten Wert, während sie in Versuch 1, wo das Harneiweiss aufs intensivste auftrat, in der oben genannten Phase gegenüber dem zuletzt genannten Wert am grössten abnahm.

Nach 2 Stunden sank sie, abgesehen von Versuch 2, in anderen Fällen (Versuch 1, 3 und 4) unter die Anfangsgrösse ab.
Fig. 2. Veränderung der zirkulierenden Blutmenge nach Bluttransfusion bei Kantharidinkaninchen (Versuch 4).

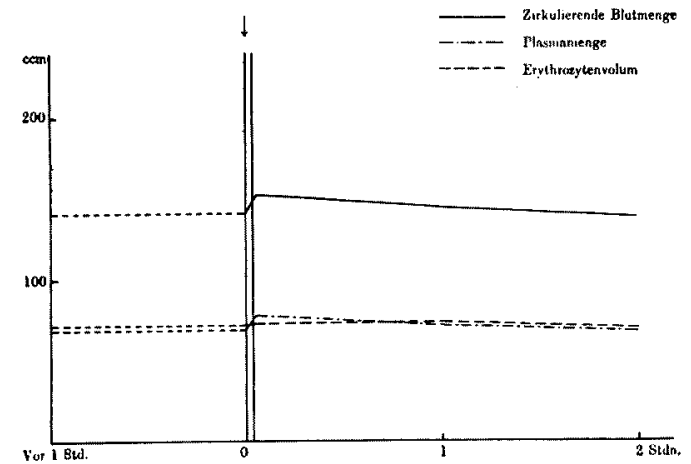


Die Plasmamenge erfulır gleich nach Bluttransfusion eine $\mathrm{Zu}$ nahme auf durchschnittlich $7,2 \%(0,1-13,9 \%)$. Nach einer Stunde wechselten Fälle, in denen die Plasmamenge mehr als der Vorwert vermehrt gefunden wurde, mit den Fällen, wo sie mehr als dieser abnahm, zur Hälfte. Nach 2 Stunden war sie, ausgenommen den Versuch 2, in allen übrigen Fällen vermindert als der Vorwert.

Das Erythrozytenvolum wurde in direktem Anschluss an die Bluttransfusion in den sämtlichen Fällen um durchschnittlich $11,2 \%(0,3-$ $31,1 \%$ ) vermehrt gefunden, dieser Betrag war jedoch geringer als die Zunahme bei gesunden Kaninchen. Hieraus geht unverkennbar hervor, dass auch in diesem Kantharidinversuch die Zunahme der Plasmamenge im Vergleich mit der Zunahme der Blutzellen spärlich ist, und dass also die Plasmafliissigkeit im Laufe der Bluttransfusion, wenn auch in geringerem Masse als bei gesunden Kaninchen, schon durch die Blutbahn hindurch gesickert ist. In einstündigem Ablauf stieg die Plasmamenge in 2 Fällen (Versuch 1 und 2) über den Vorwert in ziemlich grösserem Ausmasse hinaus, während sie in anderen 2 Fällen (Versuch 3 und 4) beinahe ebenso gross war wie der Vorwert. Die Plasmamenge in zweistündigem Ablauf zeigte keine nennenswert Veränderung im Vergleich mit derselben in einstündigem Ablauf.

Wie aus obigen Anführungen ersichtlich ist, wird die zirkulierende Blutmenge bei Kantharidinkaninchen, welche der Bluttransfusion unterworfen worden sind, in geringerem Masse als dieselbe bei gesunden, ebenfalls transfundierten Kaninchen vermehrt gefunden. Es sei im folgenden eine Betrachtung darüber angestellt.

Die nach Bluttransfusion auftretende Vermehrung der Plasmamenge in vorliegender Versuchsreihe erweist sich, wie oben gesehen, als weitaus geringer gegenüber der Zunah me bei gesunden Kaninchen. Wenn aber die Annahme, dass aus der Differenz zwischen der Grösse der beiderlei Plasmamengen unterschiedliche Grössen der zirkulierende Blutmenge $z$ wischen den beiden Tierarten sich ergeben könnten, zu Recht bestünde, so müsste die Vermehrung des Erythrozytenvolums, weil auch in vorliegendem Experiment die Transfusion von gleichen Blutmengen wie bei gesunden Kaninchen vorgenommen wurde, auch in gleichem Masse wie dieselbe bei gesunden Kaninchen stattgefunden haben. In Wirklichkeit aber erwies sich das Erythrozytenvolum nach Bluttransfusion bei Kantharidinkaninchen als geringer denn bei gesunden Kaninchen. Aus diesem Grund ist es durchaus nicht angezeigt, annehmen zu wollen, dass die Ursache, wodurch die nach Bluttransfusion auftretende Vermehrung der zirkulierenden Blut- 
menge bei Kantharidinkaninchen in geringerem Masse als bei gesunden Kaninchen erfolgt, einzig und allein in geringerer Zunahme der Plasmamenge liege. Als ursächliches Moment hierfür muss meines Erachtens höchstwahrscheinlich entscheidende Bedeutung dem Umstand zugeschieben werden, einem Umstand, dass allgemeine zirkulierende Gefässbahn bei mit Gewebsgift geschädigten Kaninchen, wie

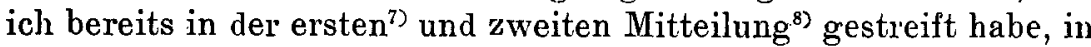
den Zustand der Verengerung versetzt ist, und infolgedessen-auch bei Blutzufuhr von aussen, wie z. B. auf dem Wege der Transfusionsich mit zugeführtem Blut in gleichem Masse, wie bei gesunden Kaninchen, anzureichen nicht mehr imstande ist. Wenn man die nach Bluttransfusion ermittelte Vermehrung der zirkulierenden Blutmenge bei jetzt in Rede stehenden Kantharidinkaninchen mit der Zunahme derselben bei gummiinfundierten Kantharidinkaninchen vergleicht, so ergibt sich, dass die erstere geringer als die letztere ist. Diese Differenz in der Zunahme zwischen beiden dürfte, wie in der zweiten Mitteilung ${ }^{87}$ geschildert, darauf beruhen, dass allgemeine Gefässbahn bei Infusion der Gummilösung durch dieselbe mehr oder minder erweitert wird, während dies bei der Bluttransfusion hingegen keineswegs nicht der Fall ist.

\section{Zusammenfassung.}

1. Wenn bei gesunden Kaninchen die Bluttransfusion von $20 \mathrm{ccm}$ pro $\mathrm{kg}$ vorgenommen worden ist, dann zeigt die zirkulierende Blutmenge in der Bluttransfusion unmittelbar nachfolgender Zeit eine deutliche Zunahme. Dieser Betrag der Zunahme ist aber geringer als die transfundierte Blutmenge. Nach der Bluttransfusion nähert sich die zirkulierende Blutmenge ganz allmählich zu früherem Niveau, sie wird jedoch nach 3 Stunden noch gewissermassen vermehrt gefunden.

Die Plasmamenge zeigt unmittelbar nach der Bluttransfusion eine Zunahme in den sämtlichen Fällen, sie rückt aber schon nach einer Stunde in der Mehrzahl der Fälle an den annähernden Vorwert heran.

Was das Erythrozyten volum nach der Bluttransfusion anbelangt, sind Zunahme desselben, welche sich unmittelbar nach der Bluttransfusion, dann in einem- zwei- und dreistündigem Ablauf ermitteln lassen, stärker als Zunahme der Plasmamenge in den entsprechenden Phasen; mit fortgeschrittenem Zeitablauf ist hier zwar die Neigung, allmählich dem fruheren Niveau zuzustreben, nachweisbar, dieser Rückgang ist aber überaus verzögert, so dass das Erythrozytenvolum 
selbst in dreistündigem Ablauf in den sämtlichen Fällen noch höhere Werte gegeüber dem Prätransfusionswert aufweist.

Es lässt sich also sagen, dass wenn einem gesunden Kaninchen Blut transfundiert worden ist, sich das transfundierte Blut, im Gegensatz zu Salz- oder Gummilösung grösstenteils an der Zirkulation beteiligt, ohne die allgemeine Gefässbahn, insbesondere die Blutdepots wesentlich $\mathrm{zu}$ beeinflussen.

2. Wenn bei Kantharidinkaninchen die Bluttransfusion von gleichen Mengen, wie bei gesunden Kaninchen, durchgeführt worden ist, nimmt die zirkulierende Blutmenge in der Transfusion unmittelbar nachfolgender Zeit auch ähnlich wie bei gesunden Kaninchen zu, der Grad der Zunahme erweist sich jedoch als geringer denn bei den letzteren. Und auch geringer ist diese Zunahme im Vergleich zu der Zunahme der zirkulierenden Blutmenge bei denjenigen Kantharidinkaninchen, denen die isotonische Gummilösung infundiert worden ist. Die Plasmamenge ist unmittelbar nach der Bluttransfusion erhöht und zwar in geringerem Masse; die Wiederherstellung zu früherem Niveau vollzieht sich so prompt, dass die Plasmamenge schon nach einer Stunde in manchen Fällen unter den Ursprungswert absinkt. Die nach Bluttransfusion auftretende Vermehrung des Erythrozytenvolums ist in den meisten Fällen geringer als bei gesunden Kaninchen.

Es lässt sich also sagen, dass wenn einem gesunden Kaninchen Blut transfundiert worden ist, sich das transfundierte Blut im Gegensatz zu Salz- oder Gummilösung grösstenteils an der Zirkulation beteiligt, ohne die allgemeine Gefässbahn, insbesondere die Blutdepots wesentlich $\mathrm{zu}$ beeinflussen. 